\title{
Mindfulness-Based Stress Reduction in the Management of Chronic Pain and Its Comorbid Depression
}

Cynthia Marske, DO; Samantha Shah, MD; Aaron Chavira, DO; Caleb Hedberg, DO, MPH; Raelin Fullmer, DO; Christopher James Clark, OMS IV; Olivia Pipitone, MPH; Paulina Kaiser, DPH

From Samaritan Health Services (Drs Marske, Shah, Chavira, Hedberg, Fullmer, and Kaiser and Ms Pipitone) in Corvallis, Oregon; and the College of Osteopathic Medicine of the PacificNorthwest at Western University (Student Doctor Clark).

Financial Disclosures: None reported.

Support: This research project was funded from a grant awarded from the Samaritan Health Services (SHS) Foundation. The grant monies were awarded via an application process directed to the Erkilla Grant for medical research. The Erkilla Grant receives private individual donations and community donations with a specific aim to fund medical research in areas of muscle-skeletal disorders and health promotion. Oversight of this

grant was provided by the SHS Internal Review Board. A midyear review was conducted

to supervise funding and research guidelines.

Address correspondence to Cynthia Marske DO, Good Samaritan Regional Medical Center, 3600 NW Samaritan Drive, Corvallis OR, 97330-3737.

Email: drmarske@msn.com Submitted July 23, 2019; revision received October 15, 2019; accepted October 28, 2019.
Context: Chronic pain (CP) is a common and serious medical condition, with an estimated 100 million people affected in the United States. In the 1990s, opioids were increasingly prescribed to manage chronic pain, and this practice contributed to the opioid epidemic of the 21 st century. To combat this epidemic, multidisciplinary approaches to chronic pain management are being researched and implemented.

Objective: To evaluate the clinical effectiveness of an 8-week mindfulness-based stress reduction (MBSR) course implemented in a semi-rural population with chronic pain.

Methods: Participants were recruited from a community-based teaching hospital in Corvallis, Oregon, for a pre-post study. Participants aged 34 to 77 years who reported having chronic pain lasting for at least 1 year before enrollment were included. Participants took an 8-week group MBSR course in 2.5-hour weekly sessions taught by an experienced MBSR instructor. Techniques were self-practiced between sessions with a goal of 30 minutes per day, 6 days per week. Pre- and postsurvey measurements of pain, depression, and functional capacity were taken via online surveys using the patient health questionnaire (PHQ-9), the Pain Catastrophizing Scale (PCS), and a shortened version of the Modified Oswestry Disability Index (MO). Participants were asked about their satisfaction with the program content, instructor, timing, and location.

Results: Twenty-eight participants were included in the study. Paired $t$ tests found significant improvements in PHQ-9, PCS, and MO percent scores from before to after the course. PHQ-9 scores decreased by a mean of 3.7 points $(95 \% \mathrm{CI},-5.5$, $-1.8)$, PCS scores decreased by a mean of 4.6 points $(95 \% \mathrm{CI}:-7.2,-2.0)$, and MO percent score decreased by a mean of $9.4 \%$ (95\% CI: $-14.2 \%,-4.6 \%)$. Results showed an overall downward shift in the distribution of depression, disability, and pain scores after the course.

Conclusions: MBSR classes were found to benefit participants with chronic pain and depression in this setting, fostering significant improvement in participant perceptions of pain, mood, and functional capacity.

J Am Osteopath Assoc. 2020;120(9):575-581. Published online August 12, 2020. doi:10.7556/jaoa.2020.096

Keywords: chronic pain, depression, mindfulness, opioids 


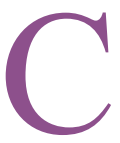

hronic pain (CP) is a common and serious medical condition, with an estimated 100 million people affected in the United States, correlating with annual costs of approximately $\$ 635$ billion. ${ }^{1}$ In the $1990 \mathrm{~s}$, the medical community responded to the growing concern about chronic pain by increasing prescriptions for opioid pharmacotherapy, which was an additive factor leading to the current opioid crisis. ${ }^{2}$ The Centers for Disease Control and Prevention has since recognized the danger of overprescribing opiates and has taken a firm stance that opioids should not be considered first-line or routine therapy for $\mathrm{CP}{ }^{3}$ This stance has inspired new efforts to take a multidisciplinary approach to the management of chronic pain and to explore nonpharmacologic treatment strategies for people with chronic pain. ${ }^{4}$

Mindfulness-based stress reduction (MBSR) is one such strategy, originally designed in the 1970s by Jon Kabat-Zinn, PhD, for patients with chronic pain (eAppendix). ${ }^{5}$ MBSR is a systematic educational program based on training people to have an awareness of the self in the present moment and a nonjudgmental manner through the intensive instruction of mindfulness meditation and mindful hatha yoga during an 8-week period. ${ }^{6}$ This technique has demonstrated measurable and long-lasting improvements in medical and psychologic well-being for various conditions including pain, depression, addiction, and anxiety. ${ }^{7-9}$ A review of 10 controlled trials ${ }^{1,6-14}$ noted that the effect of MBSR was better than passive controls but about as well as active control groups. However, these studies ${ }^{1,6-14}$ were limited by small sample size and heterogeneity. One study ${ }^{9}$ has been published comparing MBSR with other nonpharmacologic interventions that are structurally similar but do not involve mindfulness. Physiologic effects of MBSR have been seen in the regions of attention control, emotion regulation, and self-awareness through the use of functional magnetic resonance imaging. ${ }^{15-17}$ MBSR has also been shown to be effective in clinical scenarios relating to attention, depression, anxiety, stress, and addiction. ${ }^{10}$ MBSR has been shown to reduce pain effectively in a variety of studies and clinical scenarios, including lower back, upper back, shoulder, and cervical pain, as well as headaches and fibromyalgia. ${ }^{11,12,14}$

This study aimed to explore the benefits of an 8-week course on MBSR and assess whether it could successfully foster reductions in depression and pain symptoms as well as improvements in functional capacity for a semi-rural population with chronic pain in Oregon where there are issues of affordability and access to adjunctive treatment modalities. ${ }^{13}$ This study addresses pain, depression, anxiety, and stress reduction in semi-rural populations in a way that is economical and provides autonomous and flexible management options.

\section{Methods}

\section{Participants}

This study was approved by the regional institutional review board. Participants were recruited from a community-based teaching hospital through physician referral, advertising at local clinics, and word of mouth. The study was open to eligible participants who reported having chronic pain lasting for at least 1 year. Individuals were excluded if they were younger than 18 years or had pain related to cancer, terminal illness, surgery, or fractures. The study's goal was to assess adults with long-term pain deemed amenable to alternative pain management methods that were not transient, end-stage, or definitively required opioids. Accepted medical diagnoses for self-reported chronic pain included fibromyalgia, chronic back pain, chronic headache, osteoarthritis, chronic pain syndrome, and somatic pain. Participants were offered a $\$ 50$ gift card for participating in the study as compensation for their time and travel expenses.

\section{Design}

This study included 2 cohorts of MBSR classes, both of which met once per week for 2.5 hours for 8 weeks. The first cohort had 19 participants who completed the course in the fall of 2017, and the second cohort had 9 
participants who completed the course in the spring of 2018. Both cohorts were combined for this study. The course material was based on the University of Massachusetts Mindfulness-Based Practice protocol. ${ }^{5,6}$ Our protocol was modified to reduce the length of classes by removing the all-day class but included all 8 original classes of the program to allow more people to participate in the entirety of the study. ${ }^{5,6}$ Course topics focused on MBSR and were presented in a graduated weekly fashion that included a didactic and practical portion (Figure 1). The class instructor had 7 years of experience leading meditation and mindfulness courses.

Data were collected via electronic surveys 1 week before the first class session and 1 week after the final class session. The presurveys included a demographic questionnaire, the Patient Health Questionnaire (PHQ-9) $^{18}$ to measure depression, the Pain Catastrophizing Scale (PCS) ${ }^{19}$ to measure pain perception, and a shortened version of the Modified Oswestry Disability Index (MO) ${ }^{20}$ to measure disability. The postsurvey included the PHQ-9, PCS, MO, and a course evaluation questionnaire to measure participant satisfaction with the class location, timing, instructor, and content understandability and applicability. These study instruments were chosen based on ease of administration, reproducibility to other studies, and conjugate markers of the overall effect. Other surveys, such as the General Anxiety Disorder-7, were not used to prevent survey fatigue. The PHQ-9, PCS, and MO surveys were better markers of our direct question concerning management of chronic pain, depression, and functional status than other surveys.

\section{Outcome Measures}

PHQ-9 and PCS scores were calculated using published guidelines. ${ }^{18,19}$ The PHQ-9 has 9 questions and scores can range from 0 to 27 , with higher scores indicating higher depression. ${ }^{18}$ The PCS has 13 questions and scores range from 0 to 52, with higher scores indicating higher pain. ${ }^{19}$ The original MO contains 10 questions,

\begin{tabular}{|c|c|}
\hline Week 1: & $\begin{array}{l}\text { Mindfulness of the body using body scan meditation including } \\
\text { physical sensations (see eAppendix for more details). }\end{array}$ \\
\hline Week 2: & $\begin{array}{l}\text { Mindfulness of breathing using seated meditation as the } \\
\text { foundation to observe the physical sensation of the breath. }\end{array}$ \\
\hline Week 3: & $\begin{array}{l}\text { Mindfulness of the body with movement including gentle yoga } \\
\text { and stretches. }\end{array}$ \\
\hline Week 4: & $\begin{array}{l}\text { Mindfulness of the breath and body together, including formal } \\
\text { introduction of noticing unpleasant phenomena like stress/pain } \\
\text { and their manifestations in the body. }\end{array}$ \\
\hline Week 5: & $\begin{array}{l}\text { Mindfulness of sounds and thought with additional teaching on } \\
\text { noticing thoughts, sensations associated with stress/pain. }\end{array}$ \\
\hline Week 6: & $\begin{array}{l}\text { Mindfulness of speech and communication, including } \\
\text { specifically exploring how stress and somatic pain affect the } \\
\text { way we communicate. }\end{array}$ \\
\hline Week 7: & $\begin{array}{l}\text { Loving kindness meditation to develop kindness, acceptance, } \\
\text { compassion to complement and support mindfulness. }\end{array}$ \\
\hline Week 8: & $\begin{array}{l}\text { Loving kindness and mindfulness explored together to support } \\
\text { and develop a personally tailored meditation practice by each } \\
\text { participant. }\end{array}$ \\
\hline
\end{tabular}

Figure 1.

Mindfulness-based stress reduction (MBSR) curriculum by week. Course topics focused on MBSR and were presented in a graduated weekly fashion that included a didactic and practical portion.

but to reduce the burden for participants, this study only included 5 questions that covered pain intensity, self-care, sleeping, social life, and employment/homemaking. ${ }^{20}$ MO percent scores were calculated using published guidelines, but the total possible score was adjusted from 50 to $25 .^{20}$ MO percent scores range from $0 \%$ to $100 \%$, with higher scores indicating higher functional disability. ${ }^{20}$

\section{Statistical Analysis}

Participants who completed both the pre- and postsurvey and who attended at least 6 out of the 8 weekly sessions were included in the analysis. Scores had normal distribution (assessed using the AndersonDarling test) and equal variance across groups (assessed with Levene test). Paired $t$ tests were used to compare PHQ-9, PCS, and MO percent scores from before the MBSR course to after. ${ }^{18-20}$ Boxplots were created to visualize the distribution of scores 
before and after the course. This analysis was adequately powered $(80 \%)$ to detect a medium effect size (Cohen $d=0.55$ ) at $\alpha$ level of .05. Descriptive statistics were used to summarize participant satisfaction and experience. The software $\mathrm{R}$ version 3.5.1 (CRAN) was used for analysis.

\section{Results}

Of the original participants $(\mathrm{N}=38), 5$ never attended a class and were excluded. An additional 5 participants were excluded because they missed more than 3 out of 8 classes $(n=4)$ or had missing survey data $(n=1)$. Therefore, 28 participants were included in the analysis (Table 1). Participants were predominantly women (21 [75\%]), white (24 [86\%]), college graduates (21 [75\%]), and currently retired (17 [61\%]) with a mean age of 59 years. Paired $t$ tests found significant improvements in PHQ-9 (Cohen $d=0.77$ ), PCS (Cohen $d=0.68$ ), and MO (Cohen $d=0.76$ ) percent scores from before to after the course (Table 2). PHQ-9 scores decreased by 3.7 points ( $95 \% \mathrm{CI},-5.5,-1.8)$, PCS scores decreased by 4.6 points $(95 \% \mathrm{CI},-7.2,-2.0)$, and MO percent score decreased by $9.4 \%(95 \% \mathrm{CI}$, $-14.2 \%,-4.6 \%$ ). Boxplots showed an overall downward shift in the distribution of scores from before to after the course (Figure 2).

All participants agreed that the class instructor was knowledgeable and that the information on mindfulness was presented in a clear and easy to follow manner. Fifteen respondents $(50 \%)$ agreed that the information on chronic pain was clear and easy to follow, 11 were neutral (32\%) and 7 disagreed (18\%). Twenty-nine respondents $(89 \%)$ felt the information on movement was clear and easy to follow, 2 (7\%) were neutral, and 2 (4\%) disagreed. Twenty-one respondents (64\%) agreed that this program helped them better understand their pain, 10 (29\%) were neutral, 2 (7\%) disagreed. Most $(29 ; 89 \%)$ respondents agreed that this program helped them find ways to better cope with their pain (4 [11\%] were neutral).

\section{Table 1. \\ Demographic Characteristics of Participants Who Completed a Mindfulness-Based Stress Reduction Course to Manage Chronic Pain (N=28)}

\begin{tabular}{|c|c|}
\hline Demographics & $\mathbf{n}^{\mathbf{a}}$ \\
\hline Gender & \\
\hline Men & $6(21)$ \\
\hline Women & $21(75)$ \\
\hline Other or prefer not to answer & $1(4)$ \\
\hline \multicolumn{2}{|l|}{ Race/ethnicity } \\
\hline Not Hispanic or Latino & $27(96)$ \\
\hline Hispanic or Latino & $1(4)$ \\
\hline White or Caucasian & $24(86)$ \\
\hline Asian or Asian-American & $2(7)$ \\
\hline $\begin{array}{l}\text { American Indian or Alaska Native \& White or } \\
\text { Caucasian }\end{array}$ & $1(4)$ \\
\hline Mixed Race & $1(4)$ \\
\hline \multicolumn{2}{|l|}{ Highest education } \\
\hline Some elementary, middle, or high school & $1(4)$ \\
\hline High school graduate or GED & $1(4)$ \\
\hline Some college or trade/technical school & $5(18)$ \\
\hline College graduate or more & $21(75)$ \\
\hline \multicolumn{2}{|l|}{ Employment status } \\
\hline Full-time & $3(11)$ \\
\hline Part-time & $2(7)$ \\
\hline Not employed & $6(21)$ \\
\hline Retired & $17(61)$ \\
\hline Age, ${ }^{b}$ mean & 59 \\
\hline
\end{tabular}

a Data given as No. (\%) unless otherwise specified.

b Minimum age 34 years, maximum age 77 years.

Abbreviation: GED, general education development.

\section{Discussion}

This study examined the combined benefit of MBSR on patients with chronic pain and depression. As osteopathic physicians, we understand the crucial importance of treating the entire patient; the first tenet of osteopathic medicine is that a person is a unit of body, 
Table 2.

Changes in Outcome Measures From Pre- to Postcourse After Participation Mindfulness-Based Stressed Reduction Course From Paired $T$ Tests, $(\mathbf{N}=28)$

\begin{tabular}{lcccccc}
$\begin{array}{l}\text { Outcome Measure } \\
\text { Scores }\end{array}$ & $\begin{array}{c}\text { Precourse, } \\
\text { Mean (SD) }\end{array}$ & $\begin{array}{c}\text { Postcourse, } \\
\text { Mean (SD) }\end{array}$ & $\begin{array}{c}\text { Mean } \\
\text { Change }\end{array}$ & $\begin{array}{c}95 \% \text { CI for } \\
\text { Change }\end{array}$ & $\begin{array}{c}\text { P value } \\
\text { (Cohen } \boldsymbol{d})\end{array}$ \\
\hline PHQ-9 & $8.1(4.9)$ & $4.4(4.2)$ & -3.7 & $(-5.5,-1.8)$ & $<.001$ & 0.77 \\
\hline PCS & $12.6(7.2)$ & $8.0(8.5)$ & -4.6 & $(-7.2,-2.0)$ & .001 & 0.68 \\
\hline MO, \% & $26.3(16.9)$ & $16.9(16.5)$ & -9.4 & $(-14.2,-4.6)$ & $<.001$ & 0.76 \\
\hline
\end{tabular}

Abbreviations: MO, Modified Oswestry Disability Index; PCS, Pain Catastrophizing Scale; PHQ-9, Patient Health Questionnaire.

mind, and spirit. ${ }^{21}$ Our study suggests that there may be a benefit to MBSR to reduce self-reported pain and depression, which addresses the whole patient.

Compared with other studies, ${ }^{1,8-13}$ our results found similar outcomes, though we had a small number of participants. This finding helps expound on the current literature and suggests that mindfulness improves depression in those with chronic pain, which is a topic lacking in the literature.

The present study has several strengths, including common and well-validated diagnostic and assessment tools, which allows easy comparisons with existing literature. The use of a standardized MBSR curriculum helped ensure consistency in exposure to MBSR and this study's reproducibility. We have taken these results along with those from other studies to develop programs throughout the Samaritan Health Services of Lincoln, Benton, and Linn counties in Oregon. These programs offer 6 to 8 week MBSR courses in the primary care clinics and the mental health clinics to help manage chronic pain, chronic illness, and mental and behavioral health concerns as additional tools for management. MBSR has been very well received and offered alternatives to common illnesses traditionally managed with medications and other modalities.

Our study had several limitations. Selection bias was likely present in our cohort as participants with chronic pain self-selected to participate in the study. They also had to be available for weekday, daytime gatherings, which suggests that they had limited work schedules and may have been more likely to have severe worklimiting chronic pain. Additionally, the MBSR course's effect might be influenced by the social support aspect of the group class sessions. Response bias could have been present if participants exaggerated their symptom improvement to show their support for the instructor or the course. Furthermore, there was no control group to compare with, and the single instructor was not blinded to participants' status. The sample size was small, and several participants dropped out or chose not to participate after enrolling. Finally, there was a potential for bias derived from the token payments throughout the program.

To reproduce this 8-week course on MBSR, course facilitators must be trained in teaching meditation and familiar with the standardized MBSR curriculum developed by Jon Kabat-Zinn, PhD. ${ }^{5}$ A quiet location with an environment conducive for meditation and free of visual and auditory interruptions is ideal for participants to successfully complete the 8 -week course. Finally, research participants meeting inclusion criteria who are willing to participate can be referred by their health care providers to established MBSR courses as adjuvant therapy for chronic pain.

Future studies should recruit a larger study population with more homogeneity in the population groups. Future work should have active and passive control groups, which would require a significant outlay of resources and a population pool large enough to support recruitment efforts. 

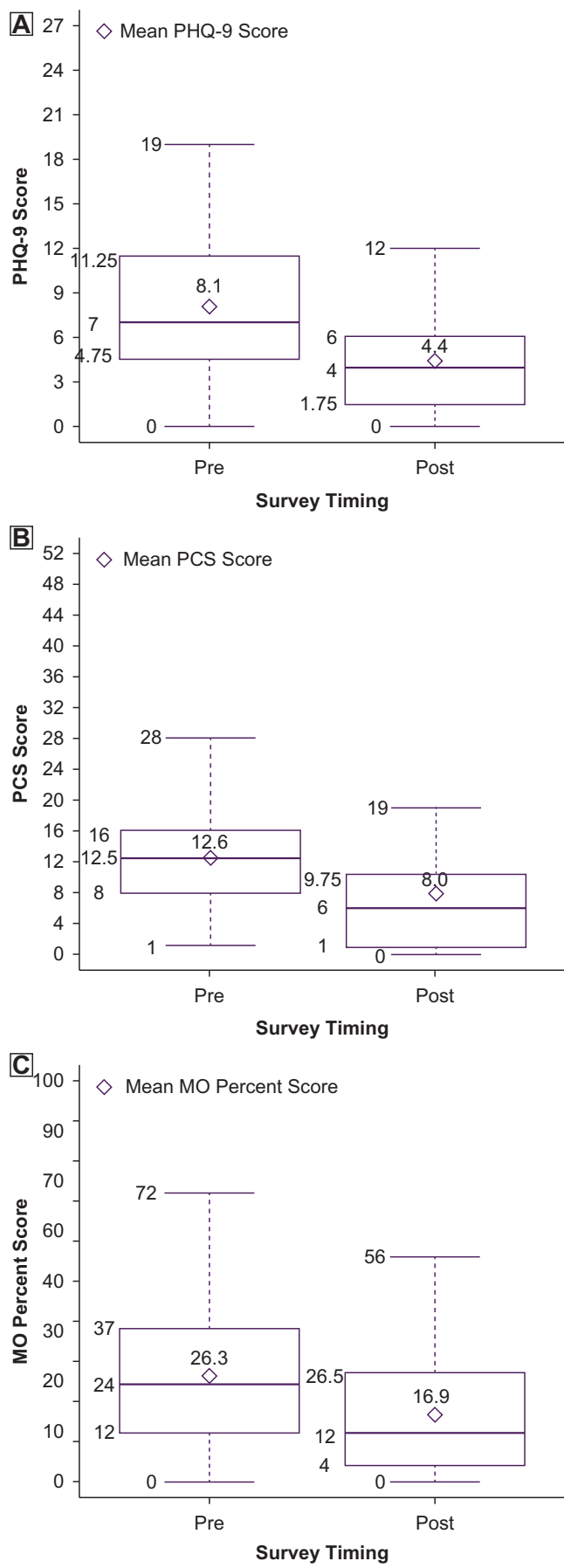

Figure 2.

Boxplots of mean (A) PHQ-9, (B) PCS, and (C) MO survey scores show an overall downward shift in the distribution of scores between pre- to postsurvey scores from participants in a mindfulness-based stress reduction course. Abbreviations: PCS, Pain Catastrophizing Scale; PHQ-9, Patient Health Questionnaire-9; MO, modified Oswestry.

\section{Conclusions}

This study suggests that MBSR classes benefitted participants with chronic pain and depression in a small, group-class setting and fostered significant improvement in participant perceptions of pain, depression, and functional capacity. This study bolsters current evidence that MBSR can be a useful adjunctive treatment for chronic pain while improving perceived depression. MBSR can be used in conjunction with pharmacologic and interventional methods to treat pain of chronic medical conditions. In this era of high prevalence of chronic pain disorders, MBSR may be a viable modality for treating patients without the use of pharmacotherapy.

\section{Acknowledgments}

We greatly appreciate the time and effort of several individuals. The course instructor, George Finch, JD, played a critical role in developing and presenting the standardized curriculum. We also appreciate the Unitarian Universalist Fellowship of Corvallis, Oregon, for supporting our research by providing a weekly meeting location. We would like to thank Good Samaritan Regional Medical Center and Samaritan Health System at large for allowing us the time, space, referrals, and resources for introduction and debriefing meetings.

\section{Author Contributions}

Drs Marske, Shah, Chavira, Hedburg, and Kaiser and Ms Pipitone provided substantial contributions to conception and design, acquisition of data, or analysis and interpretation of data; Drs Marske, Shah, Chavira, Hedburg, Fulmer, and Mr Clark drafted the article or revised it critically for important intellectual content; all authors gave final approval of the version of the article to be published; and all authors agree to be accountable for all aspects of the work in ensuring that questions related to the accuracy or integrity of any part of the work are appropriately investigated and resolved.

\section{References}

1. Leung L, Han H, Martin M, Kotecha J. Mindfulness-based stress reduction (MBSR) as sole intervention for non-somatization chronic non-cancer pain (CNCP): protocol for a systematic review and meta-analysis of randomized controlled trials. BMJ Open. 2015;5(5): e007650. doi:10.1136/bmjopen-2015-007650

2. Results From the 2013 National Survey on Drug use and Health: Summary of National Findings. Substance Abuse and Mental Health Services Administration; 2014. https://www.samhsa.gov/data/sites/ default/files/NSDUHresultsPDFWHTML2013/Web/NSDUHresults2013. pdf. Accessed July 7, 2020.

3. Dowell D, Haegerich T, Chou R. CDC guideline for prescribing opioids for chronic pain—United States, 2016. JAMA. 2016;315(15):1624-45. doi:10.1001/jama.2016.1464 
4. Tompkins DA, Hobelmann JG, Compton P. Providing chronic pain management in the "fifth vital sign" era: historical and treatment perspectives on a modern-day medical dilemma. Drug Alcohol Depend. 2017; 173(s1):S11-S21.

5. Kabat-Zinn J. An outpatient program in behavioral medicine for chronic pain patients based on the practice of mindfulness meditation: theoretical considerations and preliminary results. Gen Hosp Psychiatry. 1982;4(1):33-47. doi:10.1016/0163-8343(82) 90026-3

6. Kabat-Zinn J, Lipworth L, Burney R. The clinical use of mindfulness meditation for the self-regulation of chronic pain. J Behav Med. 1985;8 (2):163-190. doi:10.1007/BF00845519

7. Merkes M. Mindfulness-based stress reduction for people with chronic diseases. Aust J Prim Health. 2010;16(3):200-210. doi:10.1071/PY09063

8. Miller JJ, Fletcher K, Kabat-Zinn J. Three-year follow-up and clinical implications of a mindfulness meditation-based stress reduction intervention in the treatment of anxiety disorders. Gen Hosp Psychiatry. 1995;17:192-200. doi:10.1016/0163-8343(95)00025-m

9. Chiesa A, Serretti A. Mindfulness-based interventions for chronic pain: a systematic review of the evidence. J Altern Complement Med 2011;17(1):83-93. doi:10.1089/acm.2009.0546

10. Zgierska A, Rabago D, Chawla N, Kushner K, Koehler R, Marlatt A. Mindfulness meditation for substance use disorders: a systematic review. Subst Abus. 2009; 30:266-294.

11. Morone NE, Greco CM, Moore CG, et al. A mind-body program for older adults with chronic low back pain: a randomized clinical trial. JAMA Intern Med. 2016;176(3):329-337. doi:10.1001/ jamainternmed.2015.8033

12. Cherkin DC, Sherman KJ, Balderson BH, et al. Effect of mindfulness-based stress reduction vs cognitive behavioral therapy or usual care on back pain and functional limitations in adults with chronic low back pain: a randomized clinical trial. JAMA. 2016;315 (12):1240-1249. doi:10.1001/jama.2016.2323
13. Reiner K, Tibi L, Lipsitz JD. Do mindfulness-based interventions reduce pain intensity: a critical review of the literature. Pain Med. 2013;14(2):230-242. doi:10.1111/pme.12006

14. Cash E, Salmon P, Weissbecker I, et al. Mindfulness meditation alleviates fibromyalgia symptoms in women: results of a randomized clinical trial. Ann Behav Med. 2015;49(3):319-330. doi:10.1007/ s12160-014-9665-0

15. Zeidan F, Adler-Neal AL, Wells RE, et al. Mindfulness-meditationbased pain relief is not mediated by endogenous opioids. $J$ Neurosci Off J Soc Neurosci. 2016;36(11):3391-3397. doi:10.1523/ JNEUROSCI.4328-15.2016

16. Zeidan F, Martucci KT, Kraft RA, et al. Brain mechanisms supporting the modulation of pain by mindfulness meditation. $J$ Neurosci Off J Soc Neurosci. 2011;31(14):5540-5548. doi:10.1523/ JNEUROSCI.5791-10.2011

17. Tang YY, Leve LD. A translational neuroscience perspective on mindfulness meditation as a prevention strategy. Transl Behav Med. 2016;6(1):63-72. doi:10.1007/s13142-015-0360-x

18. Kroenke K, Spitzer RL, Williams SW. The PHQ-9: validity of a brief depression severity measure. J Gen Intern Med. 2001;16(9):606-613. doi:10.1046/j.1525-1497.2001.016009606.x

19. Sullivan MJL, Bishop SR, Pivik J. The pain catastrophizing scale: development and validation. Psychol Assess. 1995;7(4):524-532. doi:10.1037/1040-3590.7.4.524

20. Baker DJ, Pynsent PB, Fairbank JCT. The Oswestry Disability Index revisited: its reliability, repeatability and validity, and a comparison with the St Thomas Disability Index. In: M. Roland, J.R. Jenner (Eds.), Back Pain: New Approaches to Rehabilitation and Education. Manchester University Press;1989: 174-186.

21. Still AT. Autobiography of Andrew Taylor Still with a History of the Discovery and Development of the Science of Osteopathy. Self published;1897.

๑๑ 2020 American Osteopathic Association 